\title{
Outils de dimensionnement et d'analyse de sensibilité pour les assemblages de structures
}

\author{
Laurent Champaney ${ }^{1, a}$, Claude Blanzé$^{2}$ et Pierre Alain Boucard ${ }^{2}$ \\ 1 LEMA, Univ. de Versailles St Quentin/CNRS, 45 Av. des États-Unis, 78035 Versailles Cedex, France \\ 2 LMT Cachan, ENS de Cachan/Paris VI/CNRS, 61 Av. du Pt Wilson, 94235 Cachan Cedex, France
}

Reçu le 2 juillet 2004, accepté le 5 novembre 2004

\begin{abstract}
Résumé - L'identification des paramètres influants pour le comportement des liaisons par contact (coefficients de frottement, jeux, ...) n'est pas chose aisée. La mesure de tels paramètres est bien souvent fortement entachée d'erreurs très importantes. Les phénomènes mis en jeux (contact et frottement) sont fortement non-linéaires. La construction par simulations numériques de réponses moyennes de l'assemblage ou bien de surfaces de réponse nécessite un grand nombre de calcul très coûteux. Des techniques efficaces pour l'obtention rapide de telles informations sont proposées dans cet article. Pour décrire les assemblages nous utilisons une décomposition de type sous-structures et interfaces. L'algorithme de résolution utilisé est basé sur la méthode LATIN. Pour construire à moindre frais la surface de réponse, nous utilisons un résultat de calcul obtenu préalablement pour un jeu de paramètres comme initialisation pour un calcul avec un autre jeu de paramètres. Dans un second temps, nous proposons une méthode de construction des caractéristiques stochastiques de la réponse de l'assemblage, par projection de la solution dans une base de type chaos polynomial.
\end{abstract}

Mots clés : Assemblages / contact / frottement / méthode LATIN / incertitudes / chaos polynomial

Abstract - Advanced tools for design and sensitivity analysis of assemblies of structures. The identification of the governing parameters of the behaviour of connections including contact (friction coefficients, gaps, ...) is difficult. The measured value of such parameters is often very uncertain. Moreover contact and friction behaviours are strongly non-linear. The numerical determination of mean responses of the assembly or of response surfaces requires a large number of costly computations. This paper proposes efficients techniques for a quick determination of such informations. The assembly is described by a decomposition into substructures and interfaces, and resolution scheme used is based on the LATIN method. The proposed approach for the calculation of response surfaces is the re-using of the solution computed for a set of parameters for the initialisation of the iterative computation for a new set. This procedure is really easy with the LATIN method. The second proposed technique is the construction of the stochatic caracteristics of the response of an assembly by projection of the solution in a polynomial chaos basis.

Key words: Assemblies / contact / friction / LATIN method / uncertainties / polynomial chaos

\section{Introduction}

La simulation du comportement mécanique d'assemblages de structures complexes est de plus en plus courante dans les bureaux d'études modernes. Les récents progrès réalisés dans le domaine du calcul par éléments finis associés aux puissances toujours croissantes des calculateurs modernes permettent de conduire des calculs incluant de plus en plus de détails relatifs à la géométrie

\footnotetext{
a Auteur correspondant :

laurent.champaney@meca.uvsq.fr
}

et au comportement mécanique d'une pièce seule. Les lois de comportement sont maintenant très évoluées et bien adaptées à la complexité du matériau (métallique, plastique ou composite) considéré. Les procédures d'identification des paramètres matériaux sont de plus en plus fiables.

Néanmoins, on constate que les principales difficultés et erreurs rencontrées sont dues à la modélisation des conditions aux limites. Dans le calcul d'une pièce seule, la prise en compte de ses interactions avec le reste de l'assemblage est trop souvent réalisée de manière grossière. 


\section{Nomenclature}

\begin{tabular}{|c|c|}
\hline$\Gamma^{E E^{\prime}}$ & Interface entre les sous-structures $E$ et $E^{\prime}$ \\
\hline$\Psi_{i}(\theta)$ & $i$ ème fonction de base du chaos polynomial \\
\hline$\sigma^{E}$ & Champ des contraintes sur la sous-structure $E$ \\
\hline$\theta$ & Événement \\
\hline$\xi(\theta)$ & Variable aléatoire normale centrée réduite \\
\hline$\underline{f}_{\mathrm{d}}$ & Champ de forces volumiques \\
\hline$\underline{F}^{E}$ & Champ d'efforts de l'interface sur la sous-structure $E$ \\
\hline$\widehat{\underline{F}}^{E}$ & Champ d'efforts de l'interface sur la sous-structure $E$, vérifiant le comportement de l'interface \\
\hline$\underline{F}_{n}^{E}$ & Champ d'efforts de l'interface sur la sous-structure $E$, vérifiant l'équilibre des sous-structures à l'itération $n$ \\
\hline$\widehat{\mathbf{f}}^{E}$ & Vecteur d'efforts nodaux de l'interface sur la sous-structure $E$, vérifiant le comportement de l'interface \\
\hline $\mathbf{f}_{n}^{E}$ & $\begin{array}{l}\text { Vecteur d'efforts nodaux de l'interface sur la sous-structure } E \text {, vérifiant l'équilibre des sous-structures à } \\
\text { l'itération } n\end{array}$ \\
\hline$\widehat{\mathbf{f}}_{i}^{E}$ & $\begin{array}{l}\text { Vecteur d'efforts nodaux de l'interface sur la sous-structure } E \text {, vérifiant le comportement de l'interface. } \\
\text { ième composante dans la base du chaos polynomial }\end{array}$ \\
\hline $\mathbf{f}_{i n}^{E}$ & $\begin{array}{l}\text { Vecteur d'efforts nodaux de l'interface sur la sous-structure } E \text {, vérifiant l'équilibre des sous-structures à } \\
\text { l'itération } n \text {. ième composante dans la base du chaos polynomial }\end{array}$ \\
\hline h & Matrice de masse de l'interface \\
\hline$k_{0}$ & Paramètre scalaire des directions de recherche \\
\hline $\mathbf{K}_{E}$ & Matrice de rigidité de la sous-structure $E$ \\
\hline $\mathbf{K}$ & Opérateur de Hooke \\
\hline $\mathbf{N}$ & Matrice des fonctions de base éléments finis \\
\hline $\mathcal{R}$ & Opérateur de comportement de l'interface \\
\hline$\underline{U}^{E}$ & Champ des déplacements sur la sous-structure $E$ \\
\hline$\underline{\dot{U}}^{E}$ & Champ des vitesses sur la sous-structure $E$ \\
\hline $\mathbf{u}$ & Vecteur des déplacements nodaux \\
\hline$\underline{W}^{E}$ & Champ des déplacements à l'interface du côté de la sous-structure $E$ \\
\hline$\dot{\underline{W}}^{E E^{\prime}}$ & Discontinuité des vitesses sur l'interface $\Gamma^{E E^{\prime}}$ \\
\hline$\dot{W}^{E}$ & Champ des vitesses à l'interface du côté de la sous-structure $E$ \\
\hline$\widehat{W}^{E}$ & $\begin{array}{l}\text { Champ des déplacements à l'interface du côté de la sous-structure } E \text {, vérifiant le comportement de l'inter- } \\
\text { face }\end{array}$ \\
\hline$\underline{W}_{n}^{E}$ & $\begin{array}{l}\text { Champ des déplacements à l'interface du côté de la sous-structure } E \text {, vérifiant l'équilibre des sous-structures } \\
\text { à l'itération } n\end{array}$ \\
\hline${\widehat{\dot{W}^{E}}}^{E}$ & Champ des vitesses à l'interface du côté de la sous-structure $E$, vérifiant le comportement de l'interface \\
\hline$\underline{\dot{W}}_{n}^{E}$ & $\begin{array}{l}\text { Champ des vitesses à l'interface du côté de la sous-structure } E \text {, vérifiant l'équilibre des sous-structures à } \\
\text { l'itération } n\end{array}$ \\
\hline$\widehat{\dot{\mathbf{w}}}^{E}$ & $\begin{array}{l}\text { Vecteur des vitesses nodales à l'interface du côté de la sous-structure } E \text {, vérifiant le comportement de } \\
\text { l'interface }\end{array}$ \\
\hline$\dot{\mathbf{w}}_{n}^{E}$ & $\begin{array}{l}\text { Vecteur des vitesses nodales à l'interface du côté de la sous-structure } E \text {, vérifiant l'équilibre des sous- } \\
\text { structures à l'itération } n\end{array}$ \\
\hline$\widehat{\mathbf{w}}_{i}^{E}$ & $\begin{array}{l}\text { Vecteur des déplacements nodaux à l'interface du côté de la sous-structure } E \text {, vérifiant le comportement } \\
\text { de l'interface. } i \text { ème composante dans la base du chaos polynomial }\end{array}$ \\
\hline $\mathbf{w}_{i n}^{E}$ & $\begin{array}{l}\text { Vecteur des déplacements nodaux à l'interface du côté de la sous-structure } E \text {, vérifiant l'équilibre des } \\
\text { sous-structures à l'itération } n \text {. ième composante dans la base du chaos polynomial }\end{array}$ \\
\hline
\end{tabular}


Pour palier à ces difficultés, la plupart des industriels se tourne vers la modélisation complète de tous les composants d'un assemblage et de leurs interactions. Le problème de modélisation des conditions aux limites est alors reporté vers celui de la modélisation du comportement mécanique des liaisons entre pièces.

La modélisation par éléments finis d'assemblages de structures complexes demande de surmonter un nombre important de difficultés :

- les géométries considérées dans le calcul par éléments finis sont bien souvent directement issues de la CAO de l'assemblage et prennent généralement en compte un grand nombre de détails géométriques. Il en résulte que les modèles éléments finis manipulés sont de très grande taille et que leur traitement nécessite des puissances de calcul très importantes;

- les techniques d'assemblages (vissage, boulonnage, rivetage, soudage par point, collage, ...) nécessitent de prendre en compte des conditions unilatérales dans le comportement des liaisons en pièces. Ces conditions introduisent des non-linéarités très fortes (de type contact ou de type frottement sec) dans le comportement mécanique des assemblages. Le traitement de ce type de non-linéarité reste très délicat et nécessite l'utilisation de méthodes de résolution adaptées. Les surcoûts en temps de calcul dus à l'introduction de conditions unilatérales dans le comportement d'un assemblage tridimensionnel complexe sont très importants ;

- la modélisation du comportement des liaisons nécessite l'introduction de paramètres structuraux de natures très diverses : raideurs, coefficients de frottement ou d'adhérence, énergies de rupture, paramètres d'écrouissage, ... L'identification de ces paramètres est très difficile car elle ne peut se faire que par des essais sur structures (sur des assemblages en l'occurrence). Cette identification ne peut bien souvent se faire que par l'intermédiaire d'un dialogue entre essais délicats et calculs coûteux. Les procédures d'identification sont entachées de nombreuses erreurs de mesure ou de calcul. Les paramètres structuraux sont alors obtenus avec une marge d'incertitude très forte et difficilement quantifiable;

- la prise en compte des incertitudes sur le comportement des liaisons dans des calculs par éléments finis sur des assemblages est bien souvent indispensable [1]. On cherche alors à obtenir des réponses moyennes de l'assemblage ou mieux des surfaces de réponse lorsque les paramètres varient dans une plage importante. En utilisant des techniques de type Monte Carlo, l'obtention de ces surfaces de réponse nécessite un nombre de calculs coûteux très important. La méthode des perturbations [2-4] permet un gain de coût de calcul appréciable lorsque les amplitudes des incertitudes sont faibles. Pour des amplitudes plus grandes, on peut se tourner vers des approches de type Éléments Finis Stochastiques [5] mais les coûts de calcul restent très importants. On trouvera dans [6] un bilan des techniques existantes.
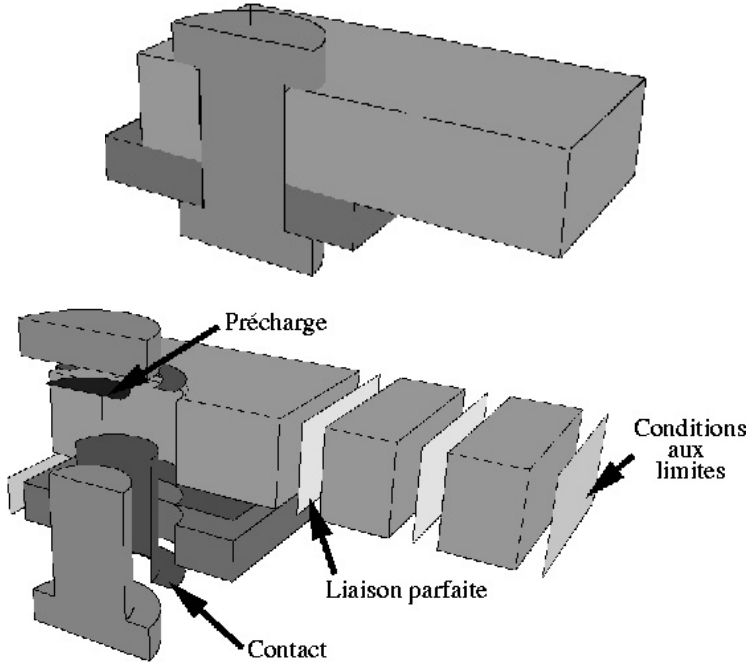

Fig. 1. Décomposition de l'assemblage.

Dans les travaux présentés ici, des méthodes spécialisées sont proposées pour répondre aux problèmes posés ci-dessus en tirant parti du fait que les nonlinéarités et/ou les incertitudes sont localisées dans les liaisons. Une représentation unifiée de ces zones de liaisons sous forme d'interfaces est utilisée à partir d'une décomposition de l'assemblage en sous-structures.

L'algorithme de résolution (la méthode LATIN [7]) utilisé permet un découplage du traitement des nonlinéarités et/ou incertitudes locales du traitement des problèmes déterministes linéaires globaux. Cela permet une très forte réduction des coûts de calcul.

Nous présentons rapidement ici la méthode utilisée et son application à la construction de surfaces de réponse pour des problèmes d'assemblages avec contact et frottement puis à la prise en compte d'incertitudes pour des assemblages collés.

\section{Approche adaptée aux calculs d'assemblages}

Pour décrire les assemblages, une décomposition de type sous-structures et interfaces est utilisée [8]. Les interfaces modélisent les liaisons entre les composants de l'assemblage. Ce sont les éléments clés de la modélisation. Elles sont considérées comme des entités mécaniques à part entière avec leur propre comportement (liaison complète, contact, frottement, ...) et leurs propres inconnues (vitesses et efforts).

L'algorithme de résolution employé est issu de la méthode LATIN proposée par Ladevèze [7]. L'idée de base de la méthode LATIN est de séparer les problèmes linéaires globaux des problèmes non-linéaires locaux. L'algorithme itératif a la particularité de traiter l'ensemble du chargement à chaque itération. On obtient ainsi une succession d'approximations de la solution sur tout l'intervalle de temps. 


\subsection{Problème sur les sous-structures}

Le problème à résoudre sur une sous-structure est : Trouver l'histoire du champ de déplacement $\underline{U}^{E}(M, t)$ et du champ de contrainte $\sigma^{E}(M, t)$ de la sous-structure $\Omega_{E}$ tels que :

- Conditions de liaison $: \forall M \in \partial \Omega_{E}$ et $\forall t \in[0, T]$,

$$
\underline{\dot{U}}^{E}(M, t)=\underline{\dot{W}}^{E}(M, t)
$$

- Équilibre $: \forall \underline{\dot{U}}^{\star} \in \mathcal{U}^{[0, T]}$ et $\forall t \in[0, T]$,

$$
\begin{aligned}
\int_{\Omega_{E}} \operatorname{Tr}\left(\sigma^{E}(M, t) \epsilon\left(\underline{\dot{U}}^{\star}\right)\right) \mathrm{d} \Omega_{E}-\int_{\Omega_{E}} \underline{f}_{\mathrm{d}} \underline{\dot{U}}^{\star} \mathrm{d} \Omega \\
-\int_{\partial \Omega_{E}} \underline{F}^{E}(M, t) \underline{\dot{U}}^{\star} \mathrm{d} S=0
\end{aligned}
$$

où $\mathcal{U}^{[0, T]}$ est l'ensemble des champs de vitesses à énergie finie sur $\Omega_{E}$ et $\underline{\dot{U}}^{\star}$ un champ de vitesses virtuelles. $\underline{f}_{\mathrm{d}}$ est un champ de forces volumiques données.

- Comportement : $\forall M \in \Omega_{E}$ et $\forall t \in[0, T]$,

$$
\sigma^{E}(M, t)=\mathbf{K} \epsilon\left(\underline{U}^{E}(M, t)\right)
$$

où $\mathbf{K}$ est l'opérateur de Hooke.

Le problème écrit ci-dessus est mal posé : des conditions en vitesses et en efforts sont imposées en même temps sur le bord du domaine. Cette surabondance de données sera levée par l'emploi d'une direction de recherche liant les efforts et les vitesses dans l'algorithme de résolution.

\subsection{Problème sur les interfaces}

Le problème à résoudre sur une interface est : Trouver les champs d'efforts $\left(\underline{F}^{E}(M, t)\right.$ et $\left.\underline{F}^{E^{\prime}}(M, t)\right)$ et de vitesses $\left(\underline{\dot{W}}^{E}(M, t)\right.$ et $\left.\underline{\dot{W}}^{E^{\prime}}(M, t)\right)$ de part et d'autre de l'interface tels que :

$\forall M \in \Gamma^{E E^{\prime}}$ et $\forall t \in[0, T]$,

- Équilibre :

$$
\underline{F}^{E}(M, t)+\underline{F}^{E^{\prime}}(M, t)=0
$$

- Comportement :

$$
\underline{F}^{E}(M, t)=\mathcal{R}\left(\underline{\dot{W}}^{E E^{\prime}}(M, \tau), \tau \in[0, t]\right)
$$

où le comportement est écrit sous forme d'une relation d'évolution éventuellement non-linéaire entre les efforts et $\underline{W}^{E E^{\prime}}$ qui est le saut de vitesses à l'interface défini de la manière suivante :

$$
\underline{\dot{W}}^{E E^{\prime}}=\underline{\dot{W}}^{E^{\prime}}-\underline{\dot{W}}^{E}
$$

Par exemple, une liaison complète entre deux sousstructures est caractérisée par le comportement suivant :

$$
\underline{\dot{W}}^{E E^{\prime}}(M, t)=0
$$

Le problème posé sur les interfaces est indéterminé : il y a quatre champs inconnus pour deux équations seulement (équilibre et comportement). Cette indétermination sera levée par l'emploi de deux équations supplémentaires de direction de recherche dans l'algorithme de résolution présenté au paragraphe suivant.

\subsection{Algorithme LATIN}

Pour la résolution du problème, on utilise un algorithme de type LATIN [7]. La solution cherchée est un ensemble de processus-champs définis sur chacune des sousstructures et des interfaces connectées :

$$
\begin{aligned}
& s= \sum_{E} s^{E} ; \\
& s^{E}=\left\{\underline{U}^{E}(M, t), \sigma^{E}(M, t), \underline{\dot{W}}^{E}(M, t), \underline{F}^{E}(M, t)\right\} \\
& t \in[0, T]
\end{aligned}
$$

Cette approche est basée sur une séparation des difficultés de manière à éviter la simultanéité du caractère non-linéaire et du caractère global du problème. Les équations à satisfaire (Éqs. (1) à (5)) sont séparées en deux groupes :

- l'ensemble $\mathcal{A}_{\mathrm{d}}$ des solutions $s$ qui vérifient les équations linéaires (et éventuellement globales) relatives aux sous-structures (Éq. (1) à (3)).

- L'ensemble $\Gamma$ des solutions $s$ qui vérifient les équations

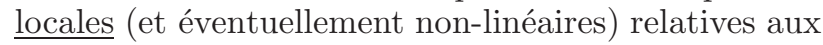
interfaces (Éqs. (4) et (5)).

La recherche de la solution du problème (qui est l'intersection des deux ensembles) se fait de manière itérative en construisant des solutions $s$ qui vérifient alternativement les deux groupes d'équations. Ainsi, une itération en composée de deux étapes :

Étape locale $: s_{n} \in \mathcal{A}_{\mathrm{d}}$ connu, trouver $\widehat{s}$ tel que :

$$
\begin{aligned}
\widehat{s} & \in \Gamma \quad \text { (sous-structures) } \\
\widehat{s}-s_{n} & \in E^{+} \quad \text { (direction de recherche) }
\end{aligned}
$$

$\underline{\text { Étape linéaire }}: \widehat{s} \in \Gamma$ connu, trouver $s_{n+1}$ tel que :

$$
\begin{aligned}
s_{n+1} & \in \mathcal{A}_{\mathrm{d}} \quad \text { (sous-structures) } \\
s_{n+1}-\widehat{s} & \in E^{-} \quad \text { (direction de recherche) }
\end{aligned}
$$

Ici, le comportement des sous-structures étant linéaire, la solution interne (en déplacement $\underline{U}^{E}(M, t)$ et en contrainte $\left.\sigma^{E}(M, t)\right)$ d'une sous-structure peut être complètement définie à partir de la solution sur les bords $\left(\underline{W}^{E}(M, t)\right.$ et $\left.\underline{F}^{E}(M, t)\right)$ : cet ensemble des champs d'efforts et de vitesses de part et d'autre des interfaces sera donc appelé, par la suite, solution du problème.

Les directions de recherche choisies sont celles pour lesquelles la convergence de l'algorithme est assurée [7]. 


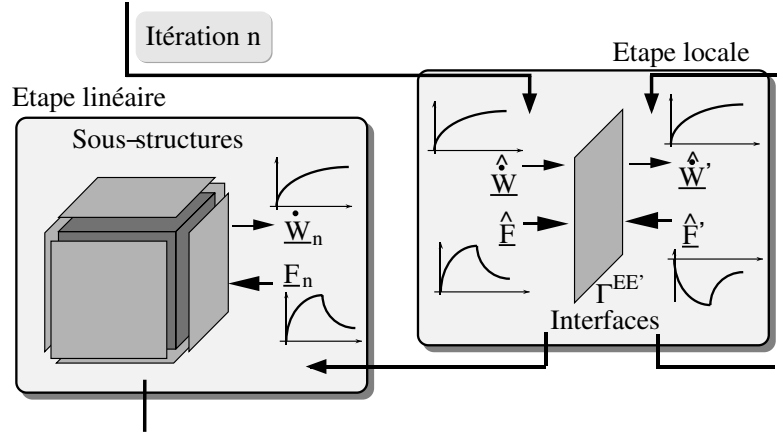

Fig. 2. Schématisation de l'algorithme LATIN.

Elles sont conjuguées et dépendent du paramètre scalaire positif $k_{0}$ :

$$
\begin{gathered}
\widehat{s}-s_{n} \in E^{+} \equiv\left(\underline{\widehat{F}}^{E}-\underline{F}_{n}^{E}\right)=k_{0}\left(\widehat{\hat{\dot{W}}}^{E}-\underline{\dot{W}}_{n}^{E}\right) \\
s_{n+1}-\widehat{s} \in E^{-} \equiv\left(\underline{F}_{n+1}^{E}-\underline{\widehat{F}}^{E}\right)=-k_{0}\left(\underline{\dot{W}}_{n+1}^{E}-\underline{\hat{W}}^{E}\right)
\end{gathered}
$$

Le processus itératif peut être schématisé comme sur la figure 2.

\subsection{Discrétisation et résolution}

On utilise une discrétisation « éléments finis » standard du déplacement sur les sous-structures :

$$
\underline{U}=\mathbf{N}^{t} \mathbf{u}
$$

Sur les interfaces, on utilise une discrétisation compatible pour les vitesses:

$$
\widehat{\dot{\hat{W}}}^{E}=\mathbf{N}^{t} \widehat{\dot{\mathbf{w}}}^{E} \quad \text { et } \quad \underline{\dot{W}}_{n}^{E}=\mathbf{N}^{t} \dot{\mathbf{w}}_{n}^{E}
$$

Le choix des directions de recherche (Éqs. (12) et (13)) nous conduit à choisir la même discrétisation pour les efforts interfaces que pour les vitesses :

$$
\underline{\underline{F}}^{E}=\mathbf{N}^{t} \widehat{\mathbf{f}}^{E} \quad \text { et } \quad \underline{F}_{n}^{E}=\mathbf{N}^{t} \mathbf{f}_{n}^{E}
$$

À l'étape locale, les problèmes de contact et de frottement sont résolus directement sur les inconnues nodales. Ainsi, la représentation utilisée pour les efforts (Éq. (16)) joue un rôle de régularisation pour le problème de frottement de Coulomb.

À l'étape linéaire l'équation d'équilibre (Éq. (2)) dans laquelle sont prises en compte les conditions de liaison (Éq. (1)), de comportement (Éq. (3)) et la direction de recherche (Éq. (13)) s'écrit :

$$
\begin{aligned}
\int_{\Omega_{E}} \operatorname{Tr}\left(\mathbf{K} \epsilon\left(\underline{U}_{n}^{E}\right) \epsilon\left(\underline{\dot{U}}^{\star}\right)\right) \mathrm{d} \Omega_{E}= \\
\int_{\partial \Omega_{E}}\left(\underline{\widehat{F}}^{E}-k_{0}\left(\underline{\dot{U}}_{n}^{E}-\underline{\widehat{\dot{W}}}^{E}\right)\right) \dot{\dot{U}}^{\star} \mathrm{d} S
\end{aligned}
$$

$$
\begin{array}{r}
\int_{\Omega_{E}} \operatorname{Tr}\left(\mathbf{K} \epsilon\left(\underline{U}_{n}^{E}\right) \epsilon\left(\underline{\dot{U}}^{\star}\right)\right) \mathrm{d} \Omega_{E}+\int_{\partial \Omega_{E}} k_{0} \underline{\dot{U}}_{n}^{E} \underline{\dot{U}}^{\star} \mathrm{d} S= \\
\int_{\partial \Omega_{E}}\left(\underline{\widehat{F}}^{E}+k_{0} \underline{\widehat{\dot{W}}}^{E}\right) \underline{\dot{U}}^{\star} \mathrm{d} S
\end{array}
$$

Après discrétisation l'équation d'équilibre (18) s'écrit :

$$
k_{0} \mathbf{h} \dot{\mathbf{u}}(t)+\mathbf{K}_{E} \mathbf{u}(t)=\mathbf{h}\left(\widehat{\mathbf{f}}^{E}(t)+k_{0} \widehat{\dot{\mathbf{w}}}^{E}(t)\right)
$$

avec :

$$
\mathbf{h}=\int_{\partial \Omega_{E}} \mathbf{N}^{t} \mathbf{N} \mathrm{d} s \text { et } \mathbf{K}_{E}=\int_{\Omega_{E}} \mathbf{B}^{t} \mathbf{K} \mathbf{B} \mathrm{d} \Omega
$$

$\mathbf{K}_{E}$ est la matrice de rigidité classique de la sous structure. $\mathbf{h}$ est un terme bord relatif aux interfaces. Il est important de remarquer que ces deux opérateurs sont constants. Ils sont indépendants de l'itération et du comportement des interfaces. Le terme $\mathbf{h}$ à un rôle régularisateur pour le problème.

Pour la résolution, on utilise un schéma d'intégration purement implicite :

$$
\mathbf{u}_{t+1}^{E}=\mathbf{u}_{t}^{E}+\Delta t \dot{\mathbf{u}}_{t+1}^{E}
$$

ce qui donne pour l'équilibre :

$$
\begin{aligned}
\left(k_{0} \mathbf{h}+\Delta t \mathbf{K}_{E}\right) & \dot{\mathbf{u}}_{t+1}^{E}= \\
& \mathbf{h}\left(\widehat{\mathbf{f}}^{E}(t+1)+k_{0} \widehat{\hat{\mathbf{w}}}^{E}(t+1)\right)-\mathbf{K}_{E} \mathbf{u}_{t}^{E}
\end{aligned}
$$

avec les conditions initiales $\mathbf{u}_{0}^{E}=0$. Puis les termes bords sont calculés à chaque instant :

$$
\begin{gathered}
\dot{\mathbf{w}}_{n}^{E}=\dot{\mathbf{u}}_{t}^{E} \operatorname{sur} \partial \Omega_{E} \\
\mathbf{f}_{n}^{E}=\widehat{\mathbf{f}}^{E}-k_{0}\left(\dot{\mathbf{w}}_{n}^{E}-\widehat{\mathbf{w}}^{E}\right)
\end{gathered}
$$

Seuls les termes de vitesse et d'efforts sur le bord sont à sauvegarder pour les itérations suivantes.

Remarque : lorsqu'on traite un problème de statique (sans intervention du temps) les variables cinématiques discrètes sur les interfaces sont les déplacements $\mathbf{w}_{n}^{E}$ et $\widehat{\mathbf{w}}^{E}$. Le problème discret à résoudre se réduit à :

$$
\left[k_{0} \mathbf{h}+\mathbf{K}_{E}\right] \mathbf{u}=\mathbf{h}\left(\widehat{\mathbf{f}}^{E}+k_{0} \widehat{\mathbf{w}}^{E}\right)
$$

avec les mêmes expressions pour $\mathbf{h}$ et $\mathbf{K}_{E}$.

\subsection{Intérêts}

En conclusion, chaque itération de l'algorithme de résolution se compose de deux étapes :

- l'étape locale concerne la résolution de problèmes non-linéaires indépendants sur chaque point de discrétisation de l'interface. Ces problèmes traduisent les comportement des interfaces et dépendent donc des paramètres de la liaison représentée par l'interface. 
- L'étape linéaire concerne la résolution de problèmes linéaires indépendants pour chaque sous-structure. En utilisant une discrétisation de type éléments finis, les systèmes linéaires générés possèdent des matrices constantes au cours des itérations et indépendantes du comportement des interfaces. Par ailleurs, cette étape donne, à chaque itération, une approximation de la solution sur tout l'intervalle de temps étudié.

Ces particularités de l'approche LATIN permettent une forte réduction des coûts de calcul pour les problèmes d'assemblages même pour des chargements statiques. Des exemples présentant les réductions de coûts de calcul obtenues pour des assemblages de structures tridimensionnelles incluant de nombreuses zones de contact sont détaillés dans [8]. Pour les problèmes présentant des incertitudes sur les paramètres de liaison, l'intérêt de l'utilisation de l'approche LATIN est double :

- les problèmes linéaires coûteux sont indépendants des paramètres de liaison. Les opérateurs associés sont construits et factorisés une fois pour toutes.

- Les approximations successives de la solution obtenues itérativement pour un jeu de paramètres de liaison peuvent être une approximation initiale non dénuée de sens pour un calcul avec un nouveau jeu de paramètres.

Ces deux caractéristiques de l'approche proposée conduisent très naturellement à deux techniques efficaces pour l'étude des assemblages présentant des incertitudes de liaison. Ces deux techniques sont présentées dans les sections 3 et 4 .

L'approche telle qu'elle est proposée ici est limitée au cas des sous-structures à comportement élastique déterministe. C'est cet aspect spécialiste qui permet une grande efficacité en terme de coûts de calcul. Une extension au cas des structures visco-plastiques déterministes 2D est proposée dans [9].

\section{Construction de surfaces de réponse}

\subsection{Principe}

Pour obtenir la surface de réponse d'un assemblage lorsque les paramètres de liaisons varient, la technique étudiée ici propose un balayage incrémental systématique de tous les paramètres dans une gamme de variation fixée par l'utilisateur. Lorsque le nombre de paramètres est grand, cela peut conduire à un très grand nombre de calculs et donc à des coûts de calcul prohibitifs.

L'idée de base de l'approche proposée est la réduction de ces coûts par la réutilisation de la solution d'un calcul obtenue pour un jeu de paramètres pour initialiser un nouveau calcul itératif pour un autre jeu de paramètres [10]. Cette procédure peut se faire très facilement avec la méthode LATIN présentée dans le paragraphe précédent. Un premier calcul est mené jusqu'à convergence avec un premier jeu de paramètres de liaisons. Pour passer à l'étude d'un nouveau jeu de paramètres, il s'agit simplement de modifier les valeurs de

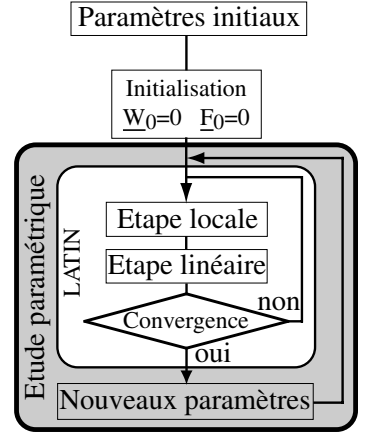

Fig. 3. Schématisation de l'étude paramétrique

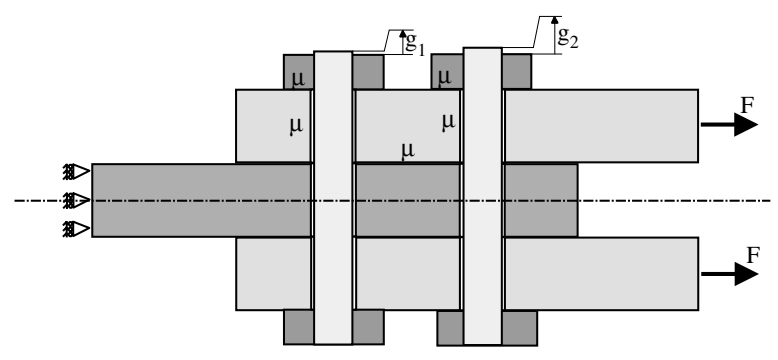

Fig. 4. Géométrie de l'assemblage boulonné.

ces paramètres intervenant à l'étape locale et de poursuivre les itérations. Ainsi, le nouveau calcul est initialisé par la solution convergée du précédent calcul et il utilise les mêmes opérateurs à l'étape linéaire.

Le processus de l'étude paramétrique avec la méthode LATIN peut être schématisé comme sur la figure 3.

Lorsque les réponses obtenues pour les deux jeux de paramètres sont proches, l'initialisation par la solution du calcul précédent permet une réduction importante du coût du deuxième calcul. Le gain reste encore très appréciable lorsque, à cause du comportement fortement non-linéaire de l'assemblage, les réponses sont plus éloignées.

Cette technique de multi-résolution par la méthode LATIN a déjà été utilisée avec succès dans différents domaines d'application $[11,12]$.

\subsection{Exemple}

Dans cette section, un exemple d'assemblage tridimensionnel est présenté. Il s'agit d'une liaison mécanique entre trois plaques, assurée par deux boulons précontraints (Fig. 4).

La figure 5 présente les caractéristiques géométriques de l'assemblage. Les boulons et les plaques sont composés du même matériau (Module d'Young $E=20000 \mathrm{MPa}$ et coefficient de Poisson $\nu=0,3$ ). La précharge des boulons est simulée par un déplacement axial relatif du corps et de la tête de chaque boulon. Les deux déplacements relatifs sont notés $g_{1}$ et $g_{2}$ (Fig. 4). Le coefficient de frottement est le même sur toutes les surfaces de contact. Il est noté $\mu$.

Dans l'étude paramétrique proposée, on étudie l'influence de la précharge de chacun des boulons et du coefficient de frottement sur la transmission des efforts dans 


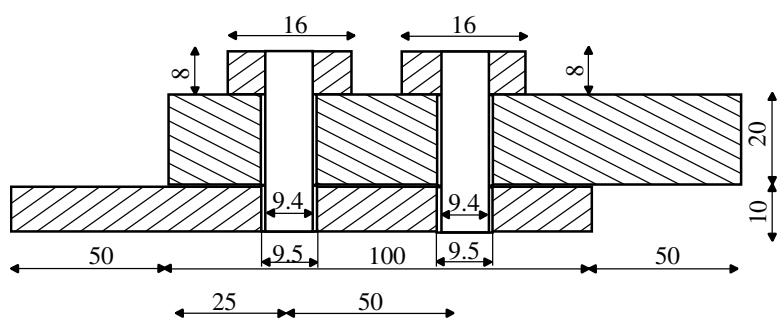

Fig. 5. Dimensions de l'assemblage boulonné.

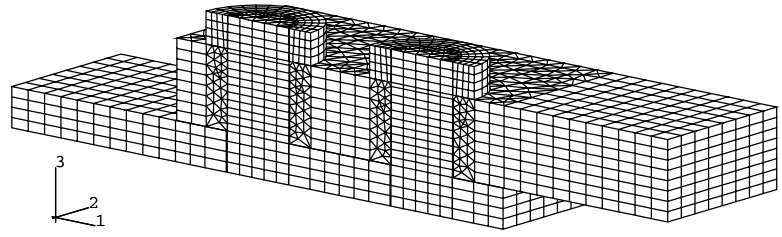

Fig. 6. Maillage de l'assemblage - 10705 éléments 8090 nœuds.

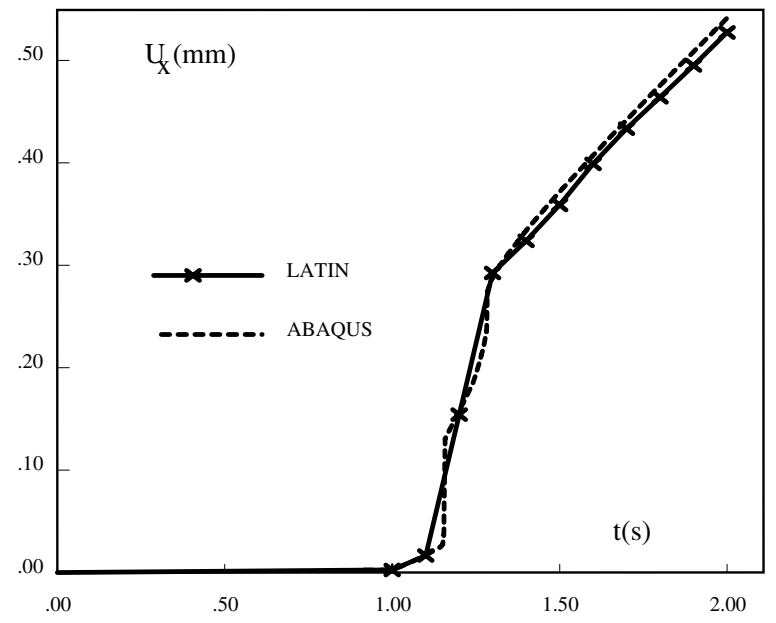

Fig. 7. Comportement global de l'assemblage.

l'assemblage. Il y a donc trois paramètres différents : un coefficient de frottement $(\mu)$ et un paramètre de précharge dans chaque boulon $\left(g_{1}\right.$ et $\left.g_{2}\right)$. Le coefficient de frottement $\mu$ peut prendre 9 valeurs différentes (de 0,1 à 0,5 par pas de 0,05$)$. Les paramètres de précharge peuvent chacun prendre 12 valeurs différentes (de $0,05 \mathrm{~mm}$ à $0,3 \mathrm{~mm}$, par pas de $0,025 \mathrm{~mm}$ ). Ainsi, pour l'étude paramétrique complète, 1296 calculs distincts doivent être effectués.

Le même maillage a été utilisé pour tous les calculs. Il est représenté sur la figure 6 . Il est composé de 10705 éléments finis linéaires (pyramides ou briques) et 8090 noeuds. Le nombre total de degrés de liberté est donc de 24270 . Ce nombre de degrés de liberté ne tient pas compte des éventuelles variables additionnelles (multiplicateurs de Lagrange, ...).

Le calcul est mené en deux étapes (step) :

- step 1 : précharge des boulons : (durée $1 \mathrm{~s}-1$ pas de temps demandé),

- step 2 : application de la charge (durée 1 s - 10 pas de temps demandés).
Tableau 1. Coûts de calcul pour un jeu de paramètres.

\begin{tabular}{ccc}
\hline & ABAQUS & LATIN \\
\hline Pas de temps & 72 & 11 \\
Temps CPU (min) & 374 & 38,1 \\
Temps horloge (min) & 407 & 40 \\
\hline
\end{tabular}

Tableau 2. Coûts de calcul pour les 1296 jeux de paramètres.

\begin{tabular}{ccc}
\hline Temps horloge & $\mathrm{h}$ & jours \\
\hline Abaqus direct & 8791 & 366 \\
LATIN direct & 864 & 36 \\
LATIN multi & 168 & 7 \\
\hline
\end{tabular}

Pour montrer l'efficacité de la méthode LATIN pour ce type de problème de contact avec frottement, une comparaison avec le code de calcul ABAQUS [13] a été menée sur une des configurations de l'étude paramétrique $\left(g_{1}=0,05 \mathrm{~mm}, g_{2}=0,025 \mathrm{~mm}\right.$ et $\left.\mu=0,3\right)$.

La figure 7 présente une comparaison de la réponse globale de l'assemblage (déplacement d'un point de la surface chargée). Les résultats ABAQUS et méthode LATIN sont très proches.

Pour des raisons de convergence, le solveur ABAQUS effectue plus d'incréments de calcul que ce qui est demandé (10 pour le step 1 et 62 pour le step 2). Les résultats de la comparaison sont donnés dans les tableaux 1 et 2 . Les calculs ont été effectués sur une station de travail HP-J5000 (3.5Go RAM - Processeur PA8500).

Les coûts de calcul de l'approche incrémentale classique d'ABAQUS pourraient certainement être réduits en utilisant des techniques d'utilisation des solutions déja calculées pour d'autres jeux de paramètres. On peut par exemple imaginer réutiliser des opérateurs tangents (comme proposé dans [14]) ou bien initialiser l'intégration sur un pas de temps avec l'incrément de déplacement correspondant pour un autre jeu de paramètres.

On peut remarquer que sur un seul calcul la méthode LATIN est dix fois plus efficace qu'un code de calcul classique. Cette efficacité, en terme d'encombrement mémoire et en terme de temps de calcul, a déjà été montrée en statique dans $[8,15]$.

La figure 8 résume tous les résultats de l'étude paramétrique. L'évolution de l'effort maximal transmissible avant un glissement relatif global des deux plaques est tracé pour chacune des valeurs du coefficient de frottement en fonction des deux paramètres de précharge $\left(g_{1}\right.$ et $\left.g_{2}\right)$. On peut remarquer que l'effort maximal transmissible dépend peu des précharges mais fortement du coefficient de frottement.

\section{Prise en compte de comportements aléatoires}

\subsection{Principe}

Les incertitudes des paramètres de liaison sont maintenant modélisées par une approche stochastique. Chaque 
<smiles>[13CH3][13CH]([13CH3])[13CH3]</smiles>
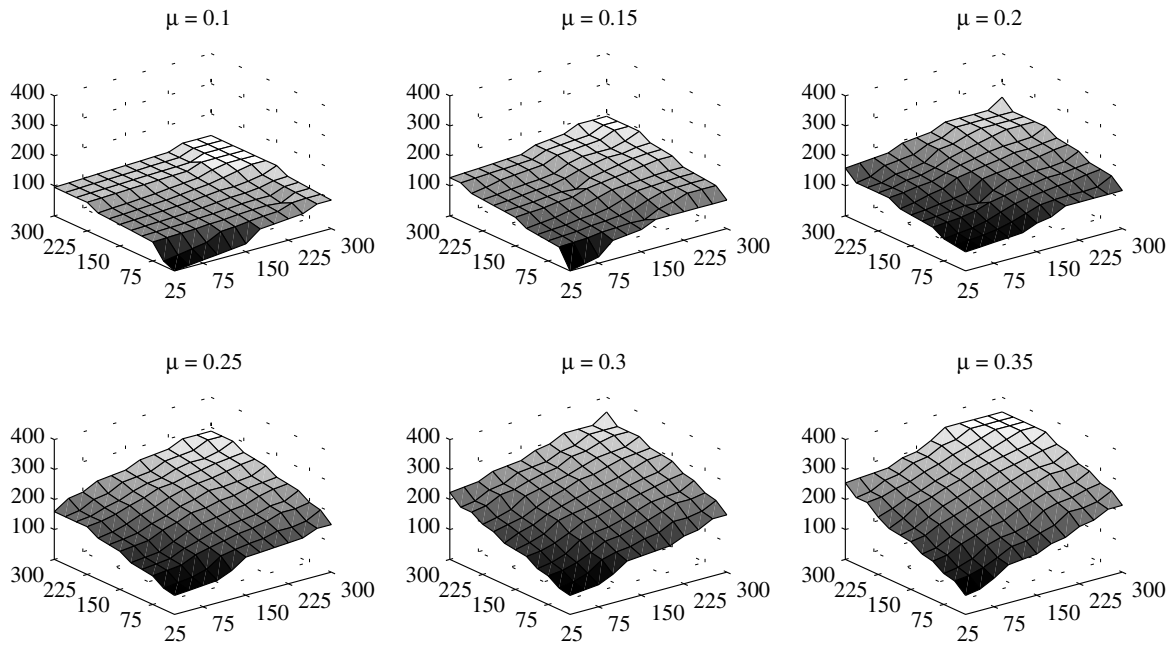

$\mu=0.4$
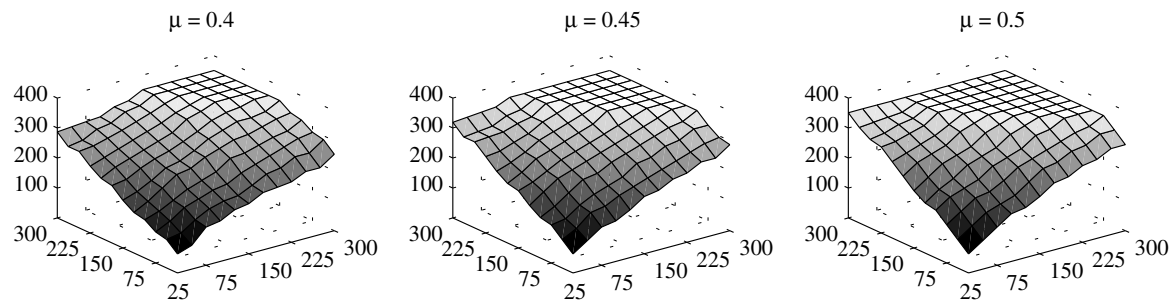

Fig. 8. Présentation condensée de tous les résultats.

paramètre d'interface (raideurs, coefficients de frottement, jeux, ...) est modélisé par une variable aléatoire :

$$
k=\bar{k}\left(1+\delta \xi_{i}(\theta)\right)
$$

où $\bar{k}$ est la valeur moyenne du paramètre, $\delta$ le coefficient de variation du paramètre et $\xi_{i}$ une variable aléatoire normale centrée réduite. $\theta$ représente la dépendance stochastique des paramètres aléatoires introduits $(\theta \in \Omega$ où $\Omega$ est l'espace des événements élémentaires).

La réponse stochastique de l'assemblage est recherchée de façon approchée. Une base de type chaos polynomial $[5,16]$ est employée :

$$
\mathbf{u}(\theta)=\sum_{i=0}^{P} \mathbf{u}_{i} \Psi_{i}(\theta)
$$

où les fonctions $\Psi_{i}(\theta)$ sont des polynômes orthogonaux des variables aléatoires normales centrées réduites $\xi_{i}(\theta)$, le produit scalaire associé étant l'espérance mathématique. Cette représentation classique des processus aléatoires a la particularité de conduire, lorsqu'elle est utilisée de manière directe, à la résolution d'un problème de taille $P+1$ fois la taille $n$ du problème déterministe. Le nombre $P+1$ de fonctions de base utilisées dépend de l'ordre de la décomposition et du nombre de variables aléatoires $\xi_{i}$. Par exemple, lorsqu'on considère 2 variables aléatoires et une décomposition d'ordre $3, P$ est égale à 10. Les coûts de calcul augmentent donc très rapidement avec l'ordre de la décomposition et le nombre de paramètres aléatoires [17].

Dans l'approche proposée ici pour réduire les coûts de calcul, seuls les paramètres de liaison sont aléatoires : le comportement des sous-structures est supposé déterministe. Lorsque cette décomposition est utilisée avec la méthode LATIN, tous les champs utilisés sont décomposés selon le même principe :

$$
\begin{gathered}
\mathbf{u}_{n}^{E}(\theta)=\sum_{i=0}^{P} \mathbf{u}_{i n}^{E} \Psi_{i}(\theta) \\
\mathbf{w}_{n}^{E}(\theta)=\sum_{i=0}^{P} \mathbf{w}_{i n}^{E} \Psi_{i}(\theta) ; \quad \widehat{\mathbf{w}}^{E}(\theta)=\sum_{i=0}^{P} \widehat{\mathbf{w}}_{i}^{E} \Psi_{i}(\theta) \\
\mathbf{f}_{n}^{E}(\theta)=\sum_{i=0}^{P} \mathbf{f}_{i n}^{E} \Psi_{i}(\theta) ; \quad \widehat{\mathbf{f}}^{E}(\theta)=\sum_{i=0}^{P} \widehat{\mathbf{f}}_{i}^{E} \Psi_{i}(\theta)
\end{gathered}
$$

Cette représentation des processus aléatoires introduite dans la méthode LATIN conduit à la résolution :

- d'un système de taille $n$ à matrice constante avec $P+1$ seconds membres, pour chaque sous-structure à chaque étape linéaire. En effet, les paramètres de liaison n'intervenant pas à l'étape linéaire, il y a découplage des fonctions de base,

- de systèmes scalaires de taille $P+1$ en chaque point de discrétisation d'une interface, à l'étape locale. En 


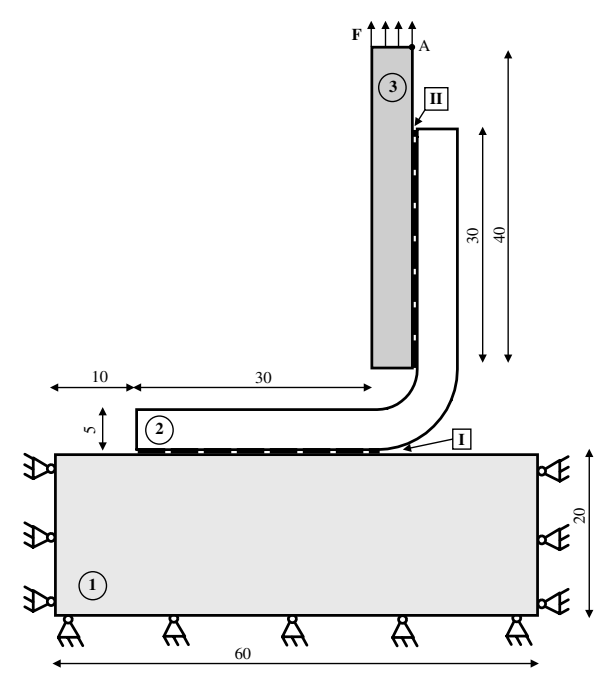

Fig. 9. Géométrie de l'assemblage collé.

effet, les paramètres aléatoires de liaison n'intervenant que dans le comportement de l'interface qui est traité localement, il n'y a qu'un couplage local des fonctions.

Les coûts de calcul peuvent donc être considérablement réduits lorsqu'on considère des systèmes de grande taille. Cela est montré dans l'exemple suivant.

\subsection{Exemple}

On considère un assemblage de trois pièces (Fig. 9) assemblées par deux zones de collage [18].

Le chargement de la structure est déterministe et représenté par une force de traction verticale sur la pièce (3). Les quantités d'intérêt sont les composantes de déplacement du point $A$ au sommet de la pièce (3) ainsi que les efforts maximum dans les joints collés.

Les joints collés (nommés I et II sur la Fig. 9) sont représentés par des interfaces. Les raideurs normales $\left(k_{\mathrm{n}}\right)$ et tangentielles $\left(k_{\mathrm{t}}\right)$ des collages, sont calculées à partir des caractéristiques des colles :

$$
k_{\mathrm{n}}=\frac{E}{e} ; \quad k_{\mathrm{t}}=\frac{E}{2(1+\nu) e}
$$

où $E$ est le module d'Young, $\nu$ le coefficient de Poisson et e l'épaisseur du joint. Les caractéristiques des matériaux sont données dans le tableau 3. Le module d'Young est indéterminé et modélisé par la loi :

$$
E(\theta)=\bar{E}(1+\delta \xi(\theta))
$$

Le maillage est composé de 17820 éléments à quatre noeuds et contient 37174 degrés de liberté.

La figure 10 donne la densité de probabilité des composantes de déplacement du point $A$ calculée avec la méthode LATIN ainsi que celle obtenue par 10000 tirages de Monte Carlo. Les résultats sont très proches et montrent l'intérêt de l'utilisation de la méthode LATIN dans ce cas.

\begin{tabular}{|c|c|c|c|}
\hline \multicolumn{2}{|c|}{ Sous-structure } & $E(\mathrm{GPa})$ & $\nu$ \\
\hline$(1)$ & & 120 & 0,3 \\
\hline (2) & & 200 & 0,3 \\
\hline (3) & & 70 & 0,3 \\
\hline Interface & $\begin{array}{c}\bar{E} \\
(\mathrm{MPa})\end{array}$ & $\nu$ & $\begin{array}{c}e \\
(\mathrm{~mm})\end{array}$ \\
\hline (I) & 500 & 0,45 & 0,3 \\
\hline (II) & 1000 & 0,45 & 0,3 \\
\hline
\end{tabular}

Tableau 3. Propriétés de l'assemblage collé.

Tableau 4. Comparaison des coûts de calcul (PC, Proc. AMD Athlon 1,4 GHz, 1,6 Go RAM).

\begin{tabular}{cc}
\hline Approche & temps CPU (s) \\
\hline Monte Carlo (10 000 tirages) & 75700 \\
Expansion Polynomiale - Direct & 780 \\
Expansion Polynomiale - LATIN & 185 \\
\hline
\end{tabular}

Le tableau 4 compare les coûts de calcul de la méthode de Monte Carlo, de la méthode spectrale directe et de la méthode LATIN. Les réductions apportées par la méthode LATIN sont très importantes pour ce type de problème.

La figure 11 montre les fonctions densité de probabilité des efforts normaux et tangentiels maximum sur l'interface (I). La construction de ces fonctions ne demande aucun effort de calcul car les efforts sont des variables de l'approche LATIN représentées par une décomposition sur le chaos polynomial. Elles sont tracées à partir de 20000 tirages de Monte Carlo sur la variable $\xi$.

Il peut aussi être intéressant de connaître l'influence de la plage de variation $\delta$ des paramètres aléatoires. La figure 12 donne l'évolution de la moyenne et de l'écart type pour la composante de déplacement $U_{y}$ du point $A$ lorsque les coefficients de variation $\delta_{\text {I }}$ et $\delta_{\text {II }}$ varient entre 0 (déterministe) et 0,4 . Le nombre de calculs à réaliser pour obtenir de telles informations par la méthode de Monte Carlo est très important. La réduction des coûts de calcul apportée par l'emploi simultané d'une projection dans une base de chaos polynomial et de la méthode LATIN est considérable. Par ailleurs, on peut imaginer, comme dans le paragraphe précédent, utiliser la solution d'un ancien calcul pour initialiser la recherche de la solution pour un nouveau jeu de coefficients de variation.

\section{Conclusions}

Nous avons présenté trois outils pour le dimensionnement et l'analyse de sensibilité dans les assemblages de structures incluant des non-linéarités et/ou des incertitudes sur les interfaces :

- une approche généraliste qui traite les problèmes déterministes de manière efficace en s'appuyant sur une décomposition de l'assemblage et sur une technique de résolution itérative (la méthode LATIN) très efficace pour ce type de problème, 

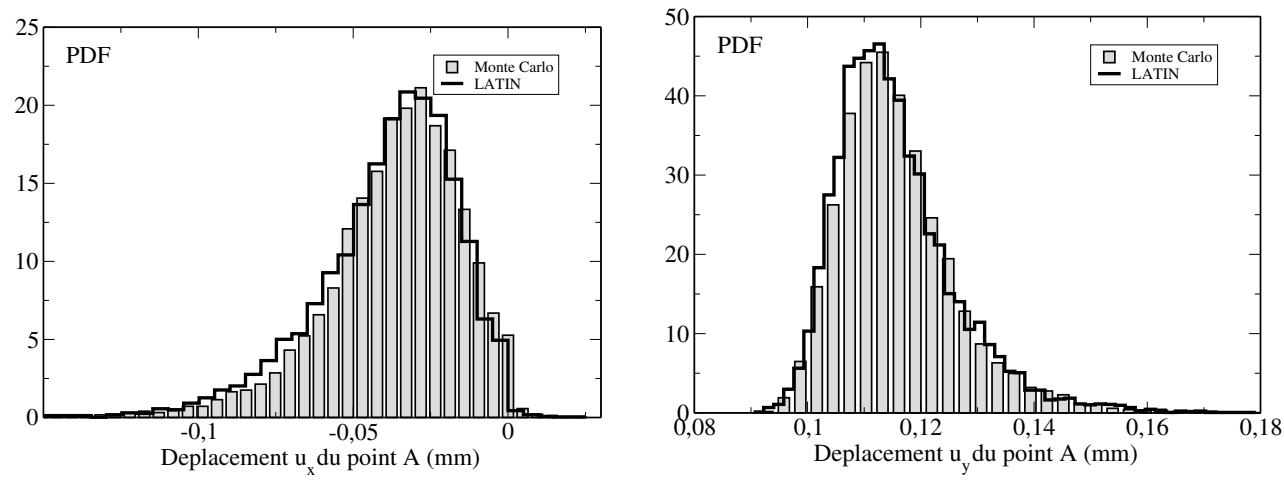

Fig. 10. Densité de probabilité des déplacements du point $A\left(\delta_{\mathrm{I}}=0,2\right.$ et $\left.\delta_{\mathrm{II}}=0,25\right)$.
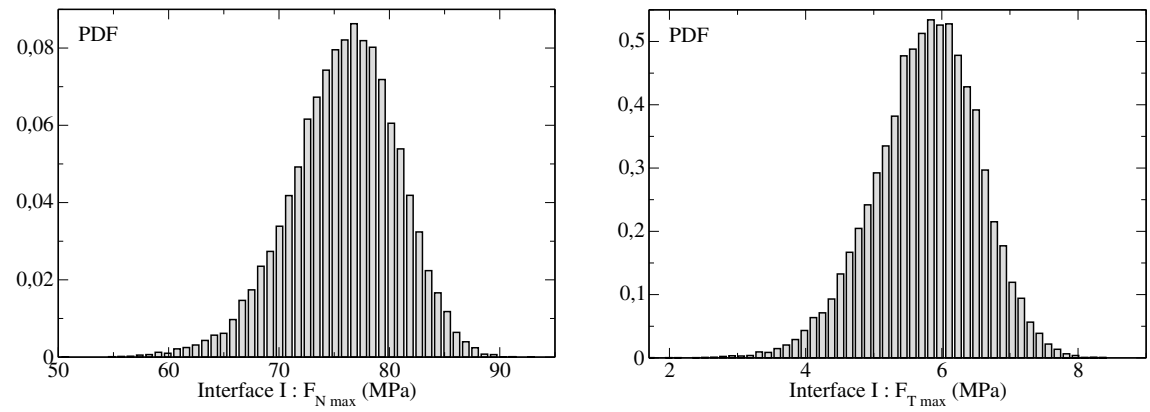

Fig. 11. Densité de probabilité des efforts normal $\left(F_{\mathrm{N}}\right)$ et tangentiel $\left(F_{\mathrm{T}}\right)$ maximum sur l'interface $I\left(\delta_{\mathrm{I}}=0,2\right.$ et $\left.\delta_{\mathrm{II}}=0,25\right)$.
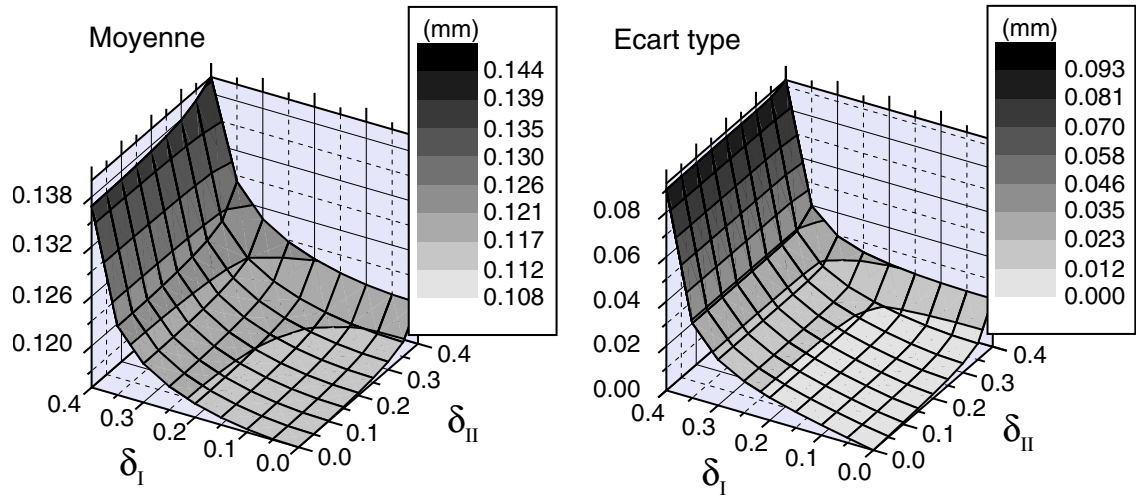

Fig. 12. Évolution de la moyenne et de l'écart type du déplacement $U_{y}$ du point $A$ en fonction des coefficients de variation $\delta_{\mathrm{I}}$ et $\delta_{\text {II }}$.

- une technique de construction rapide de surface de réponse de l'assemblage lorsque les paramètres d'interface varient. Cette technique utilise la solution d'un problème voisin pour initialiser le calcul pour un nouveau jeu de paramètres,

- une approche des problèmes d'interfaces à paramètres aléatoires par recherche de la solution dans une base de type chaos polynomial. Cette approche permet une forte réduction des coûts de calcul par rapport à une approche de type Monte Carlo ou par rapport aux techniques directes de décomposition dans le chaos.

Ces trois techniques permettent une très forte réduction des coûts de calcul pour le dimensionnement d'assemblages de structures. Cette forte réduction des coûts par rapport aux approches incrémentales classiques utilisées dans les codes de calcul industriels est bien évidemment due au fait que l'approche proposée est une approche spécialiste : elle se limite au traitement d'assemblages de structures élastiques où les seules difficultés sont les non-linéarités et les incertitudes localisées aux interfaces.

\section{Références}

[1] M. Lemaire, Fiabilité des Matériaux et des Structures, Hermès, Paris, 1998

[2] H. Benaroya, M. Rehak, Finite element methods in probabilistic structural analysis: A selective review, Applied Mechanics Rev. 41 (1998) 201-213 
[3] O.F. Macias, M. Lemaire, Éléments Finis stochastiques et Fiabilité. Application en mécanique de la rupture, Revue francaise de génie civil 1 (1997) 247-284

[4] M. Kleiber, T.D. Hien, The Stochastic Finite Element Method, Basic Perturbation Technique and Computer Implementation, John Wiley \& Sons, New York, 1992

[5] R. Ghanem, P. Spanos, Spectral techniques for Stochastic Finite Elements, Archiv. Comput. Methods Engrg. 4 (1997) 63-100

[6] B. Sudret, A. Der Kiureghian, Comparison of finite element reliability methods, Probabilistic Engineering Mechanics 17 (2002) 337-348

[7] P. Ladevèze, Non-linear Computational Structural Mechanics, New Approaches and Non-Incremental Methods of Calculation, Springer Verlag, Berlin, 1999

[8] L. Champaney, J.Y. Cognard, P. Ladevèze, Modular analysis of assemblages of 3D structures with unilateral conditions, Computers and Structures 73 (1999) 249-266

[9] P. Ladevèze, A. Nouy, On a multiscale computational strategy with time and space homogenization for structural mechanics, Computer Methods Applied Mechanics Engineering 192 (2003) 3061-3087

[10] P.A. Boucard, L. Champaney, A suitable computational strategy for the parametric study of problems with multiple contact, Int. J. Num. Meth. Engng. 57 (2003) $1259-1281$

[11] P.A. Boucard, P. Ladevèze, A multiple solution method for non-linear structural mechanics, Mechanical Engng. 50 (1999) 317-328

[12] A. Allix, P., Vidal P., A new multi-solution approach suitable for structural identification problems, Comp. Meth. Appl. Mech. Engng. 191 (2002) 2727-2758

[13] Hibbit, Karlson, Sorensen, INC. ABAQUS/Standard, version 5.8-14, theory Manual, 1998

[14] A. Legay, A. Combescure, Efficient algorithms for parametric non-linear instability analysis, Int J. Non-Linear Mech. 37 (2002) 709-722

[15] C. Blanzé, L. Champaney, P. Védrine, Contact problems in the design of a superconducting quadrupole prototype, Engineering Computations 17 (2000) 136-153

[16] R. Ghanem, Ingredient for a general purpose stochastic finite elements implementation, Comput. Methods Appl. Mech. Engrg. 168 (1999) 19-34

[17] R. Ghanem, R.M. Kruger, Numerical solutions of spectral stochastic finite element systems, Comput. Methods Appl. Mech. Engrg. 129 (1996) 289-303

[18] L. Tong, G. Steven, Analysis and Design of Structural Bonded Joints, Kluwer Academic Publisher, Dordrecht, 1999 